\title{
ENTRENAMIENTO DE LA PERCEPCIÓN ROTACIONAL CON VIDEOJUEGOS
}

\author{
Training of the rotacional perception with videogames
}

\begin{abstract}
AUTORES: CASTEJON, Marcos; CARBONELL, Xavier y FÚSTER, Héctor
\end{abstract}
Universitat Ramon Llull - Espanya

marcoscs@blanquerna.url.edu; xaviercs@blanquerna.url.edu; hectorfl@blanquerna.url.edu

\section{Resumen}

Pese a que los videojuegos son una forma de entretenimiento muy popular en el siglo 21 se subestiman las capacidades cognitivas que son capaces de poner en juego. Este estudio piloto examinó los efectos de los videojuegos en la mejora de las habilidades rotacionales y de percepción. Se utilizó el videojuego Perspective, un juego desarrollado por el DigiPen Institute of Technology que consiste en alterar un entorno 3D para resolver un puzle 2D. Los participantes jugaron un mínimo de dos horas a Perspective y respondieron el Test de matrices progresivas de Raven y el Test rotacional de Cambridge antes y después de jugar. Se hallaron diferencias significativas entre las puntuaciones obtenidas antes y después de jugar, más marcadas en el test rotacional de Cambridge. Asimismo, se observaron diferencias de género en los índices de mejora de las tareas. Tal como indican los resultados, los videojuegos son una herramienta válida para potenciar las capacidades visoespaciales y se sugiere explorar su aplicación en rehabilitación cognitiva.

\section{Palabras clave}

Videojuegos, Entrenamiento cerebral, Rotación mental, Percepción, Experimental.

\begin{abstract}
Even though videogames are a very popular form of entertainment in the $21^{\text {st }}$ century, the extent to which cognitive skills are involved is underestimated. The present pilot study experimentally examined the effects of videogames for the sharpening of perceptual and rotational skills. The videogame used was Perspective, a free-to-play game developed by DigiPen Institute of Technology that involves altering a 3D space to solve a 2D puzzle. The subjects played for at least 2 hours of Perspective, and underwent Raven's progressive matrices and Cambridge's rotational task tests before and after the exposure to said videogame. Statistically significant differences were found between the scores obtained - on both tests-before playing Perspective and afterwards. Also, gender dfferences were observed in the rate of improvement for each tasks. As shown by the results, videogames are a valid tool to enhance visuospatial abilities and its application in cognitive rehabilitation is suggested.
\end{abstract}

\section{Key words}

Videogames, Brain Training, Mental Rotation, Perception, Experimental.

\section{Introducción}

El entertraining es un término acuñado por Dyson (1990) para fusionar los conceptos de entrenamiento y entretenimiento. El entretenimiento se define como la relajación o diversión en sus múltiples facetas y depende de cada persona, por ejemplo, ver la televisión. Por otra parte, se entiende por entrenamiento la preparación, especialmente para la práctica de un deporte. En definitiva, cuando una persona entrena está más preparada pues ha potenciado sus habilidades. El entertraining consiste, por lo tanto, en una combinación de entrenamientoy entretenimiento. Algunos entrenamientos pueden ser tediosos y algunos entretenimientos pueden ser improductivos o inútiles. La propuesta del entertraining se puede definir como el entrenamiento de habilidades cognitivas y no cognitivas mediante el 
entretenimiento, por ejemplo, mediante videojuegos. Ya se ha descrito que los videojuegos tienen un gran potencial cognitivo, ya sea para uso individual o educacional y se han utilizado para potenciar habilidades de lenguaje y lectura y matemáticas, distracción de las incomodidades o ayudar con rehabilitaciones y temas de salud física y psicológica (Chatham, 2007; Griffiths, 2002; Lawrence, 1986).

El uso de videojuegos como elemento terapéutico incluye el uso de tecnología y ordenadores, y se ha demostrado son capaces de provocar un efecto positivo en el tratamiento de problemas psicológicos(Horne-Moyer, Moyer, Messer, \& Messer, 2014). Su principal ventaja consiste en, además de la eficacia demostrada, la flexibilidad que ofrecen estos, y la adaptabilidad a la terapia en todo momento, con factores como el tipo de juego, dificultad, reglas, duración y dificultad entre otros (Griffiths, 2002).

La exposición a videojuegos está relacionada con dos tipos de aprendizaje, el que viene de jugar al juego en sí, y toda aquella información que se gesta fuera del conjunto de reglas del juego, es decir, el metajuego. La clave de un videojuego que pretenda enseñar o entrenar es combinar estos dos aprendizajes para provocar el mayor impacto (Ang \& Zaphiris, 2009). Se ha explorado el uso del metajuego para enseñar nuevos idiomas y entrenar habilidades lingüísticas (Ang \& Zaphiris, 2009; Otto \& Pusack, 2009). En la revisión sistemática de Primack et al. (2012) se llegó a la conclusión de que los videojuegos mejoraron el resultado de las terapias psicológicas en un 69\% y el de las terapias físicas en un 59\%. En definitiva, existe un claro potencial para los videojuegos en el mundo de la salud. En el cuadro 1 se resumen las capacidades físicas y mentales que estimulan algunos de los géneros más populares de videojuegos: Acción, Puzle, Estrategia y videojuegos activos (Bavelier, Achtman, Mani, \& Föcker, 2012; Green, 2014; Greenfield, DeWinstanley, Kilpatrick, \& Kaye, 1994).

Cuadro 1. Efectos positivos sobre capacidades cognitivas de los videojuegos de Acción, Puzle, Estrategia y videojuegos activos

\begin{tabular}{l}
$\begin{array}{l}\text { Efectos positivos sobre capacidades cognitivas de } \\
\text { los videojuegos de Acción, Puzle, Estrategia y } \\
\text { videojuegos activos }\end{array}$ \\
\hline $\begin{array}{l}\text { Acción: Memoria visual, atención, control ejecutivo, } \\
\text { atención selectiva, distribución espacial, reflejos, } \\
\text { coordinación ojo-mano, atención visual dividida, } \\
\text { etc. }\end{array}$ \\
\hline $\begin{array}{l}\text { Puzle: Solución de problemas, pensamiento } \\
\text { abstracto, capacidad para asimilar normas, } \\
\text { memoria, etc. }\end{array}$ \\
\hline $\begin{array}{l}\text { Estrategia: Pensamiento estratégico, capacidad de } \\
\text { decisión, velocidad de respuesta, atención, } \\
\text { memoria, etc. }\end{array}$ \\
\hline $\begin{array}{l}\text { Videojuegos activos: Equilibrio, confianza, } \\
\text { reducción de emociones negativas, mejora de } \\
\text { funciones motoras y aceleración de rehabilitación. }\end{array}$ \\
\hline
\end{tabular}

Fuente: Elaboración propia a partir de (Bavelier et al., 2012; Green, 2014; Greenfield et al., 1994)

Una de estas capacidades es la potenciación de habilidades motoras, psicomotoras, mentales y de coordinación (Staiano \& Flynn, 2014). Sin embargo todavía no se han evaluado los efectos que la exposición a los videojuegos tiene sobre las capacidades visoespaciales.

El objetivo general del estudio fue observar si es posible entrenar capacidades cognitivas con el uso de videojuegos, concretamente, si es posible entrenar las capacidades visoespaciales con la exposición al videojuego Perspective. La hipótesis principal fue que la exposición al juego generará mejoras de las capacidades visoespaciales.

\section{Método}

\subsection{Participantes}

Los participantes fueron 21 hombres y 8 mujeres obtenidos mediante un muestreo por conveniencia. Los participantes tenían edades comprendidas entre los 18 y los 25 años $(M=20,69$; DT= 2,40). El 34,5\% (n $=10)$ jugaban menos de 5 horas semanales a videojuegos. Los criterios de inclusión fueron disponer de un ordenador personal capaz de ejecutar el videojuego Perspective, así como conexión a Internet. 


\subsection{Instrumentos}

Cuestionario socio-demográfico. Se creó un cuestionario ad-hoc en el que se registró edad, sexo y horas de juego semanales.

\subsection{Test de matrices progresivas de Raven}

El Test de matrices progresivas de Raven diseñado en 1936 mide la inteligencia y la habilidad mental general mediante la comparación de formas y el razonamiento por analogías y se considera una buena herramienta para medir la inteligencia deductiva (Raven, 2003). Es un test ampliamente utilizado en psicología clínica por ser totalmente visual, sencillo, no cultural (de modo que el nivel de escolaridad es irrelevante), no verbal (de modo que no importa el idioma que hable el sujeto, ni si es analfabeto o sordomudo) y se puede realizar en internet sin perder efectividad (Arce-Ferrer \& Martinez Guzman, 2009). Se aplicó la escala general del Test de Matrices Progresivas de Raven (MPG), que consiste en 5 series integrada de 12 ítems cada una. Los ítems dentro de una misma seria son progresivamente más difíciles y requieren una mayor capacidad de análisis y codificación de la información.

El Test Rotacional de la batería de Cambridge Brain Sciences, diseñada por Owen et al. (2010), mide la capacidad del cerebro para rotar objetos, ya sean bidimensionales o tridimensionales. Un claro ejemplo de esta capacidad sería orientarse en un mapa; lo más fácil es mirar al norte y poner el mapa con el norte en la parte superior, pero con una buena capacidad de rotación mental, no es necesario. Esta capacidad también sirve para orientarse en zonas difíciles, como bosques, y para crear imágenes mentales de objetos tridimensionales con mayor facilidad y precisión (Shepard \& Metzler, 1971). El test presenta dos matrices formadas por cuadrados de colores, y una de las matrices rotada por un múltiplo de 90 grados. Al rotarlas, estas matrices son o idénticas o distintas dependiendo de la posición de un único cuadro. El participante debe obtener el mayor número de puntos posible indicando si las matrices son idénticas o distintas, y resolviendo el mayor número de problemas posible en 90 segundos. Si el participante acierta, obtiene un número de puntos igual al número de cuadrados contenidos en la matriz, y el siguiente problema tendrá más cuadrados. Si el participante comete un error, se le restan tantos puntos como cuadrados integraban la matriz, y el siguiente problema presentará menos cuadrados. Las parejas generadas son aleatorias, de manera que repeticiones del test no necesariamente garantizan una mayor puntuación debido al aprendizaje previo.

\section{Procedimiento}

A los participantes se les facilitó un enlace para acceder a un formulario con el consentimiento informado, las instrucciones para participar en el estudio, la descarga del videojuego que cumplía la función de entrenamiento y el acceso a los test. Los participantes completaron la Escala General del Test de Raven y la Tarea de Rotación de Cambridge Brain Sciences. Tras esto jugaron 2 horas al videojuego Perspective. Finalmente, completaron de nuevo el Test de Raven y la Test Rotacional y anotaron las puntuaciones en el formulario.

Perspective es un juego de plataformas experimental que combina elementos 2D con elementos 3D con una mecánica especial: el control de la perspectiva (DigiPen Institute of Tecnology, 2012). El jugador controla a un personaje que debe navegar de forma bidimensional unas plataformas de colores. Estas plataformas están dispuestas, a su vez, en un espacio tridimensional. El jugador puede alternar entre el control del personaje, y el control de la cámara. Cuando el usuario controla la cámara altera la perspectiva de modo que la disposición de las plataformas varía. El usuario debe hacer uso del cambio de perspectiva para facilitar el avance del personaje a través de las plataformas.

\section{Análisis de los datos}

Para el análisis de datos se utilizó el software IBM SPSS Statistics 22. Se comprobó la normalidad de las variables implicadas en las pruebas estadísticas mediante la prueba de Shapiro-Wilk, cuyo estadístico 
ha sido identificado como la prueba de normalidad univariada recomendada independientemente del tamaño de la muestra (Razali \& Wah, 2011), así como el análisis de la distancia de Mahalanobis (DM) para identificar valores atípicos, usando como umbral $p<, 001$ (Tabachnick \& Fidell, 2007). Se realizó una regresión múltiple por pasos para determinar si el uso de videojuegos y el género afectaban a las puntuaciones iniciales del Test de Raven y el Test
Rotacional. Por último, se realizaron análisis de varianza (ANOVA) de medidas repetidas de 2x2 (Momento del test $\mathrm{x}$ Género) para comprobar el efecto del entrenamiento para el Test de Raven y el Test Rotacional. Por último, se realizaron análisis de varianza (ANOVA) de medidas repetidas de 2x2 (Momento del test $\mathrm{x}$ Género) para comprobar el efecto del entrenamiento para el Test de Raven y el Test de Rotación.

\section{Resultados}

Para analizar si el género o el tiempo dedicado a los videojuegos eran predictores de la habilidad espacial al inicio de la investigación se realizó una regresión múltiple por pasos. Tan solo el género contribuyó significativamente a explicar la varianza de la habilidad espacial al inicio del estudio tanto para el Test de Raven $\left(F(1,27)=4.51 ; p<.05 ; R_{\text {ajustada }}^{2}=\right.$ $.11)$ como para el Test Rotacional $(F(1,27)=5.58 ; p$ $\left.<.05 ; R_{\text {ajustada }}^{2}=.14\right)$.

Para determinar el efecto del entrenamiento con Perspective en el Test de Raven y Test Rotacional, se realizaron sendos ANOVA de medidas repetidas 2x2 (Momento del test $\mathrm{x}$ Género). Tal como se muestra en la Figura 2, los análisis mostraron una interacción significativa entre el momento en el que se realizó el Test de Raven y el género del participante $(\lambda=.83$; $\left.F(1,27)=28.04 ; p<.05 ; \eta^{2}=.173\right)$. Asimismo, se observó un efecto significativo del momento en que se realizó el Test Rotacional $(\lambda=.66 ; F(1,27)=$ $\left.13.91 ; p<.05 ; \eta^{2}=.34\right)$, pese a que en este caso no se observó ninguna interacción con el género de los participantes (ver Tabla 1).

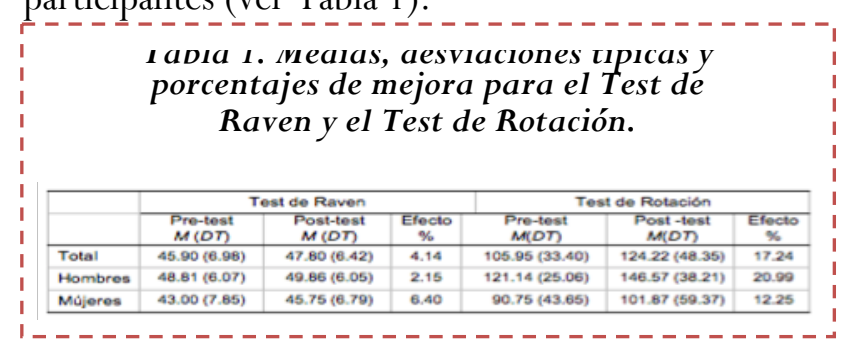

Figura 1.Efecto del entrenamiento en Perspective sobre las puntuaciones en el Test de Rave.

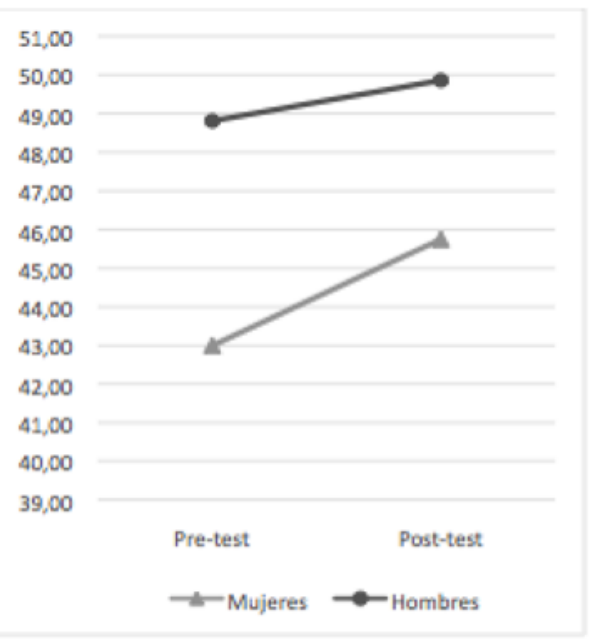

Por último, se realizó una correlación bivariada entre el nivel alcanzado en Perspective durante el entrenamiento y las puntuaciones tanto previas en el Test de Raven $(r=.77 ; p<.001)$ y el Test Rotacional $(r=.93 ; p<.001)$, como posteriores para el Test de Raven $(r=.69 ; p<.001)$ y el Test Rotacional $(r=$ $.78 ; p<.001)$.

\section{Discusión}

El objetivo de este estudio piloto fue compobar si es posible entrenar capacidades cognitivas con el uso de videojuegos. Los resultados obtenidos indican que el videojuego Perspective mejora estas habilidades. En el
Test de Raven las reducidas mejoras observadas podrían deberse a un simple efecto aprendizaje debido a la administración repetida. Con todo, existen diferencias ostensibles entre las puntuaciones 
y los índices de mejora de hombres y mujeres. Estas diferencias son recurrentes en la literatura sobre el Test de Raven (Lynn \& Irwing, 2004).

En cuanto a los resultados del Test Rotacional, se encuentra una mejora más pronunciada de las puntuaciones posteriores al entrenamiento con Perspective (17.24\%). Si bien se observaron diferencias ostensibles en las puntuaciones iniciales, estas se pueden atribuir al dimorphismo de género en lo que atañe a tareas de tipo visoespacial (Hänggi, et al., 2010; Maguire, Burgess \& O’Keefe, 1999). Con todo, las diferencias entre hombres y mujeres en lo que atañe al índice de mejora no resultaron significativas. En conjunto, esto significa que, pese a que los hombres puntúan mejor que las mujeres en la tarea rotacional antes y después del entrenamiento, tanto hombres como mujeres se benefician de igual modo del entrenamiento mediante Perspective.

La fuerte correlación positiva entre la puntuación en ambos test previo a Perspective y el nivel al que se ha llegado en el juego nos indica que el factor $G$ de la inteligencia-medido por el test de Raven-está directamente relacionado con el rendimiento en el juego y la capacidad de solucionar los puzles planteados en cada nivel. También indica que la capacidad de rotación mental está directa y fuertemente relacionada con el rendimiento en el juego y que aquellos con mejor percepción rotacional son capaces de llegar a niveles más avanzados y difíciles.

Según nuestros datos, Perspective se revela como una buena herramienta de entrenamiento de las capacidades perceptivas. El concepto de entertraining es posible en este caso y se podría utilizar Perspective como entrenamiento visoperceptivo. El entertraining es actualmente una realidad y se pueden entrenar capacidades cognitivas y físicas con videojuegos como elemento de entretenimiento (Dyson, 1990). En el caso de Perspective, el aprendizaje ocurre únicamente a través de las mecánicas de juego (es decir, manipulación de la perspectiva, movimiento, etc.), y elude cualquier tipo de transmisión pasiva de conocimiento (es decir, narrativa y storytelling). En consecuencia, estamos hablando de un entrenamiento en inteligencia fluida (el factor $\mathrm{G} f$ o habilidad eductiva) que incluye aspectos como el reconocimiento de patrones, el razonamiento abstracto y la resolución de problemas (Catell, 1987). Del mismo modo que se pueden mejorar habilidades de atención selectiva, memoria y reflejos con los juegos de acción (Bavelier et al., 2012; Boot et al., 2008), se pueden mejorar las capacidades visoespaciales con videojuegos como Perspective, que incluyen la manipulación del entorno y el uso de habilidades perceptivas como la rotación mental. Se sugiere que Perspective, u otro videojuego con características similares, podría utilizarse con fines terapéuticos, a modo de rehabilitación, para entrenar o recuperar la funcionalidad de las capacidades visoespaciales (Primack et al., 2012).

Por otra parte, cabe tener en cuenta las limitaciones de la investigación. El diseño adolece de la falta de un grupo control con el que contrastar los índices de mejora. Asimismo, sería adecuado replicar estos resultados con una muestra mayor y más homogénea de hombres y mujeres. Por último, de cara a futuros estudios, sería recomendable controlar e incluso comparar el desempeño de los participantes según la experiencia cualitativa en el uso de videojuegos. Esto quiere decir que, más allá del control de las horas de juego, el tipo de videojuego en el que tienen experiencia los participantes (es decir, diferentes géneros de videojuego), puede ofrecer una perspectiva interesante sobre la interpretación los resultados obtenidos. Más aun teniendo en cuenta que la percepción rotacional es una de las capacidades cognitivas que algunos géneros específicos de videojuegos pueden estimular (Boot, Kramer, Simons, Fabiani, \& Gratton, 2008; Green, 2014; Greenfield et al., 1994).

\section{Conclusión}

Con la suma de pruebas realizadas podemos afirmar que jugar a Perspective genera diferencias significativas en las capacidades de concentración y percepción rotacional. La inteligencia rotacional definida por Shepard y Metzler (1971) está relacionada con la capacidad de crear mapas mentales de objetos y 
posicionarse mentalmente en la perspectiva deseada, además de generar mapas mentales de lugares extensos con mayor facilidad. Al entrenar la percepción rotacional se puede mejorar la capacidad de orientación del individuo (Shepard \& Metzler, 1971). Si los resultados preliminares obtenidos en el presente estudio se reproducen de forma consistente, Perspective podría llegar a usarse en patologías como el Parkinson, no solo por su potencial para mejorar las capacidades de mapeado y orientación, sino por su valor lúdico capaz de mejorar la adhesión de los pacientes al tratamiento. Iguialmente, se sugiere que
Perspective, $\mathrm{u}$ otro videojuego con características similares, podría utilizarse en la rehabilitación cognitiva para entrenar o recuperar la funcionalidad de las capacidades visoespaciales (Primack et al., 2012). Los test utilizados miden, además de las capacidades cognitivas específicas ya mencionadas, la capacidad de concentración del individuo, y Perspective, como juego de puzles, requiere de cierto nivel de concentración para superar los niveles. Con esto en mente, futuras líneas de investigación podrían investigar el uso de Perspective o videojuegos similares en casos de TDAH (Boot et al., 2008).

\section{Referencias}

- Ang, C. S., \& Zaphiris, P. (2009). Developing enjoyable second language learning software tools: A computer game paradigm. In Handbook of Research on Effective Electronic Gaming in Education (pp. 1372-1389). doi:10.4018/978-1-59904-808-6.ch079

- Arce-Ferrer, A. J., \& Martinez Guzman, E. (2009). Studying the Equivalence of Computer-Delivered and Paper-Based Administrations of the Raven Standard Progressive Matrices Test. Educational and Psychological Measurement, 69(5), 855-867. doi:10.1177/0013164409332219

- Bavelier, D., Achtman, R. L., Mani, M., \& Föcker, J. (2012). Neural bases of selective attention in action video game players. Vision Research, 61, 132-143. doi:10.1016/j.visres. 2011.08.007

- Boot, W., Kramer, A., Simons, D., Fabiani, M., \& Gratton, G. (2008). The effects of video game playing on attention, memory, and executive control. Acta Psychologica, 129(3), 387-398. doi:10.1016/j.actpsy.2008.09.005

- Catell, R. (1987). Intelligence: Its Structure, Growth and Action: Its Structure, Growth and Action. Amsterdam: Elsevier Science Publishers.

- Chatham, R. E. (2007). Games for training. Communications of the ACM. doi: $10.1145 / 1272516.1272537$

- DigiPen Institute of Tecnology. (2012). Per- spective. Recuperado de http://games.digipen.edu/games/perspectiv e\#.VZZZvRvtlHy

- Dyson, E. (1990). Entertraining software. Forbes, 145, 136. Recuperado de http://search.ebscohost.com/login.aspx?dir ect $=$ true $\& \mathrm{db}=$ bth \&AN $=9003260293 \&$ site $=$ ehost-live

- Green, S. (2014). The perceptual and cognitive effects of action video game experience. In Learning by Playing: Video gaming in education (pp. 29-41).

- Greenfield, P. M., DeWinstanley, P., Kilpatrick, H., \& Kaye, D. (1994). Action video games and informal education: Effects on strategies for dividing visual attention. Journal of Applied Developmental Psychology, 15(1), 105-123.

- Griffiths, M. (2002). The educational benefits of videogames. Education and Health, 20, 47-51.

- Hänggi, J., Buchmann, A., Mondadori, C. R., Henke, K., Jäncke, L., \& Hock, C. (2010). Sexual dimorphism in the parietal substrate associated with visuospatial cognition independent of general intelligence. Journal of Cognitive Neuroscience, 22(1), 139155.

- Horne-Moyer, H. L., Moyer, B. H., Messer, D. C., \& Messer, E. S. (2014). The Use of Electronic Games in Therapy: a Review with Clinical Implications. Current Psychiatry Re- 
ports, 16(12). doi:10.1007/s11920-0140520-6

- Lawrence, G. H. (1986). Using computers for the treatment of psychological problems. Computers in Human Behavior, 2, 43-62. doi:10.1016/0747-5632(86)90021-X

- Lynn, R., \& Irwing, P. (2004). Sex differences on the progressive matrices: A metaanalysis. Intelligence, 32(5), 481-498.

- Maguire, E. A., Burgess, N., \& O’Keefe, J. (1999). Human spatial navigation: cognitive maps, sexual dimorphism, and neural substrates. Current Opinion in Neurobiology, 9(2), 171-177.

- Otto, S. E. K., \& Pusack, J. P. (2009). Computer-assisted language learning authoring issues. Modern Language Journal, 93, 784 801 doi: $10.1111 /$ j.15404781.2009.00973.x

- Owen, A. M., Hampshire, A., Grahn, J. A., Stenton, R., Dajani, S., Burns, A. S., ... Ballard, C. G. (2010). Putting brain training to the test. Nature, 465(7299), 775-778. doi: $10.1038 /$ nature09042

- Primack, B. A., Carroll, M. V., McNamara, M., Klem, M. Lou, King, B., Rich, M., ... Nayak, S. (2012). Role of video games in improving health-related outcomes: A sys- tematic review. American Journal of Preventive Medicine, 42, 630-638. doi:10.1016/j.amepre.2012.02.023

- Raven, J. (2003). Raven Progressive Matrices. In Handbook of nonverbal assessment (pp. 223-237). doi:10.1007/978-1-4615-01534_11

- $\quad$ Razali, N. M., \& Wah, Y. B. (2011). Power comparisons of Shapiro-Wilk , KolmogorovSmirnov, Lilliefors and Anderson-Darling tests. Journal of Statistical Modeling and Analytics, 2(1), 21-33.

- Shepard, R. N., \& Metzler, J. (1971). Mental Rotation of Three-Dimensional Objects. Science, 171, 701-703. doi:10.1126/science. 171.3972.701

- Staiano, A. E., \& Flynn, R. (2014). Therapeutic Uses of Active Videogames: A Systematic Review. Games for Health Journal, 3(6), 351-365. doi:10.1089/g4h.2013.0100

- Tabachnick, B.G., \& Fidell, L.S. (2007). Using Multivariate Statistics (5th Ed.). Boston: Pearson.

\section{Forma de Citación}

CASTEJON, Marcos; CARBONELL, Xavier y FÚSTER, Héctor: Entrenamiento de la percepción rotacional con videojuegos. Revista Communication Papers, $N^{\circ}$ 6, páginas 74 a 80. Departamento de Filología y Comunicación de la Universidad de Girona. Recuperado el _ de_ de 2 de: http://www.communicationpapers.es 